\title{
In Situ Injection Rate Measurement to Study Single and Split Injections in a Heavy- Duty Diesel Engine
}

\author{
Bassam S. E. Aljohani, Moez Ben Houidi, Rafig Babayev, Khalid Aljohani, and Bengt Johansson
}

Clean Combustion Research Center (CCRC), King Abdullah University of Science and Technology (KAUST),Thuwal 23955-6900,

Saudi Arabia

\begin{abstract}
The split injection strategy holds a potential for high pressure combustion engines. One advantage of such strategy is the capability to control the heat release rate, which also implies the use of multiple split-injections with relatively short dwell intervals. Most injection rate measurement techniques require installment of the injector on a dedicated test rig. However, these techniques fail to accurately reproduce real-engine operating conditions. Using the spray impingement method, this paper investigates the injection rate of a high flow-rate solenoid injector while being operated on the engine. The aim is to have an experimental configuration as similar as possible to the real engine in terms of the acoustics and the fuel temperature within the injection system. The assumption of spray force proportional to the spray momentum is used to measure the injection rate. The spray momentum is measured while the injector is mounted on the Volvo D13 engine and connected to the in-series fuel rail and pump. A high-natural-frequency piezoelectric pressure transducer is mounted perpendicularly at $4 \mathrm{~mm}$ from one of the nozzle holes. The injector and sensor are contained within a specially designed collector for the injected fuel, which is maintained at atmospheric pressure and temperature. Experiments with single injection are conducted varying the Duration of Injection (DOI) from 400 up to $2000 \mu$ s. The tests with split double-injections are conducted with fixed DOI of $500 \mu \mathrm{s}$ while the dwell time are varied from 100 up to $1000 \mu \mathrm{s}$. All tests are performed at the rail pressures of 500, 1000, 1500 and 2000 bar while the engine is operated at $1200 \mathrm{rpm}$. Results show that the injection rate shape of single injections is highly dependent on the rail pressure profile. With double split-injections, the rate of the second injection as well as the total fuel mass injected increases when the dwell time is shortened. Short dwell intervals boost the fuel quantities as a result of the altered needle response. Long dwell time between two equally-long injections generate similar injection rates. The injector hydraulic delay was more pronounced when dwell time was kept long enough. Overall, higher injection pressure advances the effective start of injection while retarding the effective end of injection.
\end{abstract}

\section{Introduction}

The injection rate is an important spray parameter that influences the fuel-air mixing and combustion. The appropriate control of the injection rate, and consequently the HR shape, can improve the combustion characteristic, leading to improved combustion efficiency and reduced emissions. The Euro VI and US13 emission regulations introduced significantly stricter limits on $\mathrm{NO}_{x}, \mathrm{PM}$, and unburned hydrocarbon emissions. Recent diesel injectors are designed to meet such regulations through their capability of delivering multiple injection events [1]. Nordgren et al. [2] demonstrated low NOx emission levels for early injection timing and an increase of NOx at Page 1 of 12 retarded injection timing in HCCI combustion. In addition, Nehmer and Reitz [3] studied the effect of injection strategy on soot and NOx formation in a diesel engine and demonstrated significant reductions of these emissions. Babayev et al. [4-5] also studied different injection strategies and their effects on the combustion characteristics. In particular, constant-pressure combustion, which was proven superior to the conventional diesel combustion, required complex injection strategies with multiple main injection events. Injection rate measurements have been carried out for those injection strategies and will be further discussed in the Multi-split injection subsection.

Furthermore, Wang et al. [6] quantitatively studied the instantaneous mass flow rate for each split-injection to better understand the total mass quantity per injection. They concluded that the interaction from spray-to-spray is strong function of the dwell interval and first main injection duration. Moreover, as the injection pressure increases, the start of injection is shortened. Lou et al. [7] investigated the injection rates for six-hole injector with respect to injection pressure. In their work, an adjustable stand was used to align the sensing element for each hole with respect to the spray direction and demonstrated that the quantity of injected fuel does not change significantly with varying outlet-target distance as well as the angle between the injector axes and the sensor axes, namely target-axes angle. However, with the outlettarget distances above $12 \mathrm{~mm}$, a delay was noticed on the injection start and injection end, as well as lower peak injection rate and fuel injection rate shape were reported [7].

In literature, many techniques have been introduced to characterize high-pressure injection events. The Bosch long tube [8] and Zeuch [910] methods have extensively been used to measure the injection rate. The Bosch method relies on recording of the pressure waves during an injection event. When fuel is injected into a compressible fluid (usually diesel fuel as well), pressure waves are generated, which can then be recorded by a pressure sensor. The flow rate of fuel can therefore be estimated using the recorded pressure, fluid density, speed of sound, and cross-sectional area of the Bosch tube. Zeuch's method measures the increase of pressure when a fuel is injected into a constant volume chamber filled with fuel. Thus, the change of pressure can be measured to predict the volumetric flow rate. It was observed that both techniques provide slightly different injection rate averages [11]. Payri et al. [12] proposed a method to correct the signals obtained for excessive injections using theoretical approach. Another approach of measuring the injection rate was proposed by researchers from the Tokyo Institute of Technology. They used a revolving disc, which measures unsteady fuel flow rate utilizing pressure signals [13]. Bopp et al. [14] used laser-Doppler sensor to measure the instantaneous volume flow rate of the axial spray velocity of a capillary pipe flow. This measuring technique is commonly used for measurements of low volumetric flow rate over a wide operating condition since it can capture instantaneous changes of the flow. Similarly, Zeng et al. [15] 
numerically studied the macroscopic spray characteristics, using laser technique, to study the dominating forces associated with liquid-jet breakup in a broad range of direct-injection gasoline engine conditions. The forces acting on the sensing element were found to be the inertial, viscous, surface tension, and aerodynamic drag forces. Marcic et al. [16] as well as Payri et al. [17] numerically and experimentally studied the injection rates of multi-hole injectors and hole-to-hole variations of diesel injectors. Payri et al. [18], studied the effect of cavitation on diesel spray behavior using two bi-orifices geometry for the injected mass flux and momentum flux. Desantes et al. [19], developed a theoretical model based on the boundary layer theory to characterize the discharge coefficient in a non-cavitation diesel injector.

In the aforementioned studies, the injection rates were measured while the injector is mounted on a separate test rig dedicated for this characterization. Despite all of their advantages, these experimental rigs might not fully reproduce real engine operating conditions. For instance, injection rate fluctuations are often observed in the measurements with the long-tube method. Questions can be raised about the acoustic behavior of the injectors in this type of rigs where the fuel pump, rail and pipes may be substantially different from those used in real engine. Manin et al. [20] observed that injection events can generate pressure waves in the rail, which, in turn, can propagate all the way back until the orifice of the injector nozzle. Hence, the fuel injection rate can be significantly influenced by the pressure waves. In attempt to further understand the injection rate shapes and capture these acoustic effects, we measured the spray momentum from a highpressure Diesel injector in-situ. The injector was mounted on the Volvo D13 HD engine and connected to the in-series fuel pump and rail. The engine test bench is originally modified to allow the use of optical diagnostic techniques. The setup is adapted to install a force transducer in front of one of the six-holes and measure the spray momentum at atmospheric pressure. This allowed a characterization representative of real engine operation, particularly in terms of acoustics effects on the injection rate shape. To our knowledge, this is the first study to report injection rate measurement using the impingement method on an in-series engine configuration.

In our previous studies $[4,21]$ injection rate measurements were conducted for various injection strategies including single and multiple (up to five) main injections events, required to achieve constantpressure combustion. The measurements were performed using the same experimental configuration presented in this paper. A comparison of $\mathrm{n}$-heptane with diesel fuels was investigated by AlRamadan et al. [21] using the current set-up to understand the effect of different fuels on the hydraulic delay time. It was noticed that the effective end of injection varied from one fuel to another at identical injector settings. The injection rate of diesel fuel was found to be higher than that of gasoline fuel because of the higher density of diesel fuel. It was shown that the response of the needle to energizing timings was faster for the n-heptane case compared to diesel. This was likely related to the higher viscosity of diesel fuel. A more viscous fuel exerts stronger viscous forces on the injector needle, resulting in a slower motion. This restriction causes a delay on the end of injection when using diesel-like fuels, which was also reported by Payri et al. [22]. Furthermore, an increase in the mass flow rate was observed as the injection pressures increased, while the injector hydraulic delay was shortened for both fuels. In this study, single and double-split injection strategies are investigated. The in-situ spray momentum measurement is first detailed. The test configuration is described along with force signal acquisition and post-processing. The effective start of injection is defined and corrected with respect to the distance between the force sensor and the injector nozzle. The characterization is then used to understand the behavior of the injector at different rail pressures, injection durations and dwell times.

\section{Experimental Setup}

\section{Engine Test Bench}

A spray momentum rig is designed and mounted directly on a heavyduty Volvo single cylinder diesel engine. The in-series production direct injection Volvo D13C500 engine is modified based on the Bowditch design. The cylinder head is lifted and a piston extension coupled with an optical liner are used in studies focused on optical diagnostics. Similar engine design is used in [23]. The schematic of the standard engine test bench is presented in Figure 1. In the present work, the optical parts were dismounted to allow the installation of the spray momentum rig. This method allowed an in-situ characterization of the spray injection rate which enabled to accurately capture the dynamic and acoustics behavior of the fuel injection system under more realistic engine conditions compared to conventional test rigs. The injector is characterized while the engine is operating using the in series production injection system pump, rail and pipes. The engine specifications are detailed in Table 1 . The fuel injection system is scaled for injection pressures up to 2700 bar and is composed of mainly a common rail, a six-hole F2 Delphi non-pumping injector and a pumping injector where only the pumping unit is used to pressurize the fuel.

Table 1.Specifications of the engine on which the injection rate was measured

\begin{tabular}{|l|l|}
\hline Engine Configuration & 4 stroke, Single cylinder \\
\hline Cam / Valve Configuration & SOHC / 4 valves per cylinder \\
\hline Engine Speed & $1200 \mathrm{rpm}$ \\
\hline Fuel Injection System & Delphi F2 Common Rail \\
\hline Rail Pressure Range & up to 2700 bar \\
\hline Injector Type & Solenoid Direct injection \\
\hline Number of Holes & 6 holes \\
\hline Nozzle Orifices Diameter & $\sim 240 \mu \mathrm{m}$ \\
\hline Injector Umbrella Angle & 147 degree \\
\hline Injector Static Flow Rate at 100 bar & $2.5 \mathrm{~L} / \mathrm{min}$ \\
\hline
\end{tabular}



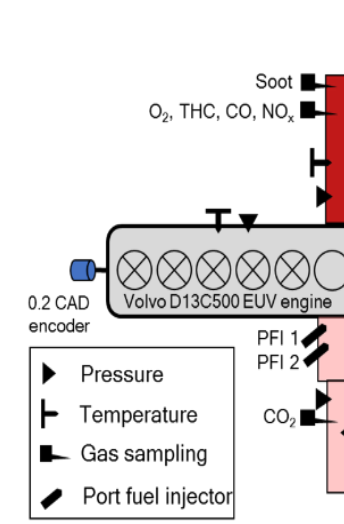

Back pressure
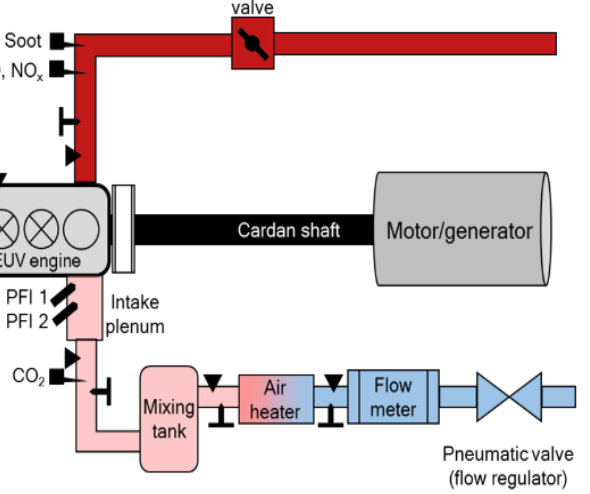

Figure 1. Schematic of the optical engine test bench.

\section{Injection Rate Test Rig}

A cylindrical container is mounted under the injector nozzle directly under the lifted cylinder head to collect the injected fuel. A piezoelectric pressure sensor (AVL GU22C, used here as a force sensor) is installed in front of one of the injector holes and positioned at $4 \mathrm{~mm}$ from the nozzle, as illustrated in Figures 2 and 3. Its sensing area is wider than that of the spray impingement. According to Luo et al. [7], the $4 \mathrm{~mm}$ distance is short enough and should induce a negligible error on the injection rate measurements. A charge amplifier is used to condition the signal from the sensor. The signal is acquired with $0.2 \mathrm{CAD}$ resolution (approximately $36 \mathrm{kHz}$ sampling frequency) using the engine control and acquisition system. This system is based on the NI CRio architecture. Simultaneously, the force signal is acquired with $5 \mu$ s time resolution (200 kHz sampling frequency) using the NI PCI-DAQ, which is trigged by the engine control system. The injection timing was set around TDC for both the single and double injection events. Approximately, 30 cycles were recorded at each test case. A high-speed camera was used to monitor the setup during the experiments. The injection rate measurements are performed with low sulfur grade diesel. The fuel collected after a counted number of tests is weighted then used for the calibration of the fuel mass flow rate. The air inside the fuel collector is maintained at atmospheric pressure and temperature. It was technically difficult to pressurize the air and apply a backpressure on the spray. Several studies [24-26] have shown that this parameter should not significantly alter the injection rate measurement, particularly at high rail pressures. No damages or erosions were noticed on the pressure sensor plate. The sensor was carefully installed perpendicularly to the spray and was stiffly maintained in its optimized position for all the set of experiments.

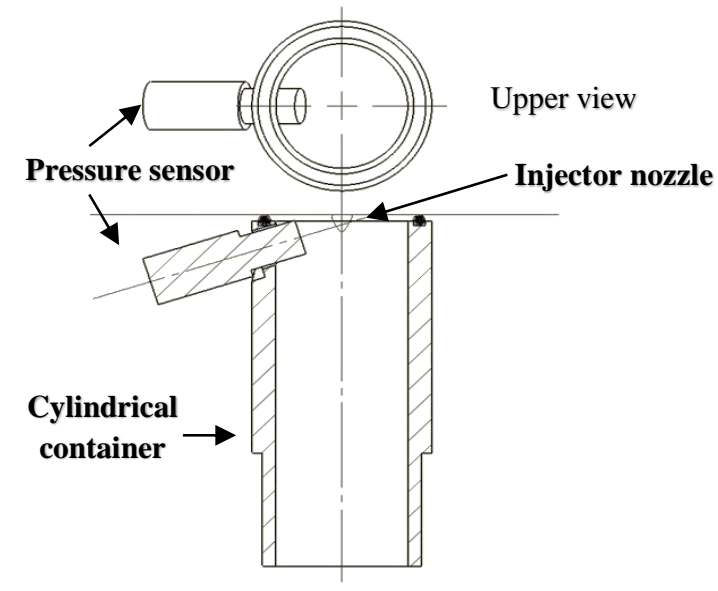

Figure 2. Overview of the sensor and nozzle positions in the injection rate test rig.

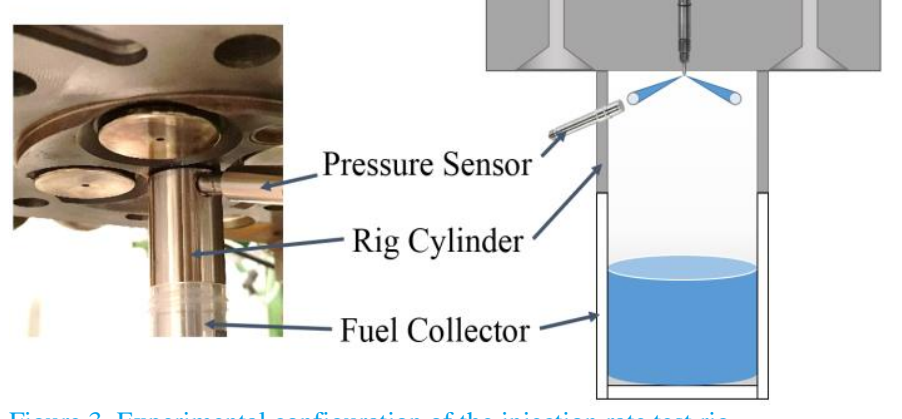

Figure 3. Experimental configuration of the injection rate test rig.

\section{Measurement methodology}

\section{Theoretical Background of spray momentum}

Based on the conservation of momentum, the fuel mass flow rate can be modeled with the following equation $[18,19]$ :

$$
\dot{m}_{f}=\sqrt{F \rho_{\text {fuel }} A_{\text {hole }}}
$$

Where,

$\boldsymbol{F}$ - Measured force.

$\boldsymbol{\rho}_{\text {fuel }}$ - Fuel Density.

$\boldsymbol{A}_{\text {hole }}$ - Cross-sectional area of the nozzle injector hole.

Since the area of the nozzle hole and the fuel density can assumed to be constant, the injection rate is considered to be proportional to the square root of the measured force.

$$
\dot{m}_{f} \sim \sqrt{F}
$$

$\dot{m}_{f}=$ calibrated fuel mass flow rate. 
Two methods are then possible to calculate the injection rate from the measured force: (i) measure quantitatively the force (with a calibrated sensor) and properly calculate the proportionality factor that depends on the nozzle holes and fuel density, (ii) measure the force and use the collected fuel to calibrate the injection rate measurement. The second method was utilized here for the measurement of the injection rate. Several studies in literature $[18,25]$ have demonstrated that both methods provide consistent results.

\section{Post-processing of the force signal}

\section{Filtering method}

The signal generated by the force sensor was amplified with a Kistler 5015A charge amplifier. A low-pass (LP) filter with a threshold of 30 $\mathrm{kHz}$ was hardware applied. The threshold is reasonably below the natural frequency of the force sensor which is $100 \mathrm{kHz}$ according to AVL specifications. The spectrum analysis (using FFT algorithm) of the raw signal with and without this filter is presented in Figure 4. Without filtering, the force signal has strong fluctuations. Most likely, the spray impingement on the sensor too fast and cause resonance vibrations in the sensing element. Thus, the use of high natural frequency sensor and the proper filtering were needed to have a better quality force signal.

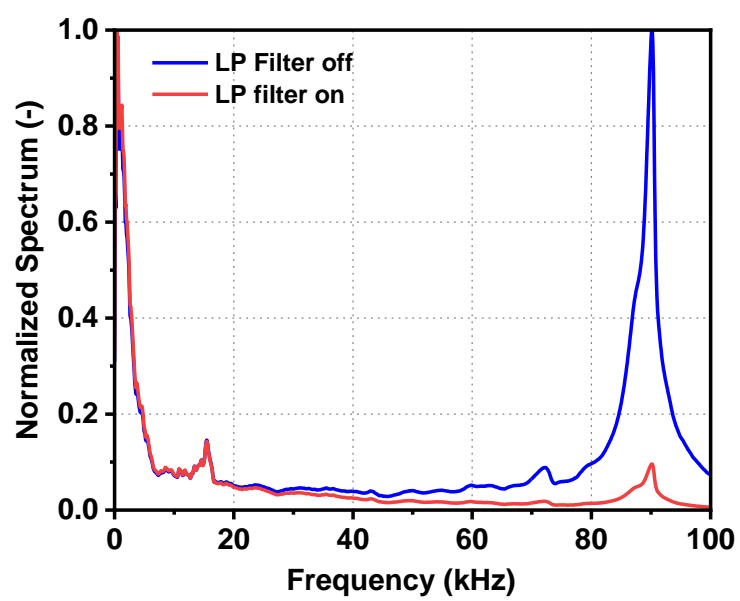

Figure 4. Spectrum analysis of the raw force signal: red line with low-pass LP filter, blue line without filter.

An additional LP (4 kHz threshold) FIR filter is applied in the postprocessing. In this process, the signal is filtered with hamming window. Following that process, the signals are pegged and converted from relative to absolute measurements.

\section{Charge leak correction}

It has been observed that the signals drop below negative values after the end of injection. This is caused by the amplifier charge leak. This phenomenon is usually electronically compensated. However, this compensation is optimized for typical in-cylinder pressures and was not perfectly adapted for the current measurements. Hence, a correction was needed to properly measure the peak of the injection rate and the effective end of injection. The following equation is used:

$$
\begin{aligned}
P_{\text {Corr }}[i+1]= & P_{\text {Corr }}[i]+\left(P_{\text {Corr }}[i+1]-P_{\text {Corr }}[i]\right) \\
& + \text { Comp. factor }(p[i] \\
& \left.-P_{\text {mean }}\right)
\end{aligned}
$$

Where,

$\boldsymbol{P}_{\text {corr }}=$ corrected pressure.

$\boldsymbol{P}=$ measured pressure.

Comp. factor $=$ compensation factor.

$\boldsymbol{P}_{\text {mean }}=$ mean measured pressure of all cycle.

\section{Noise correction and injection rate calibration}

Despite the filtering and the averaging of the force signals, very small noise fluctuations were still observed before and after the injection period. In all cases, the signal to noise ratio was higher than 250 and the noise was suppressed to allow the calculation of the square root of the force. A calibration factor was calculated from two test cases of approximately 200 consecutive cycles each. The obtained calibration factors were consistent and the average value was used to deduce the injection rate and total mass of the non-calibrated test cases. As shown in Eq. (4) and (5), the calibration factor is calculated from the measured total mass (from a counted number of injections) and the integrated non-calibrated fuel mass-flow-rate $\dot{m}_{f}^{*}$.

$$
\begin{gathered}
\alpha_{C}=\frac{m_{f}}{\int \dot{m}_{f}^{*} d(t)} \\
\dot{m}_{f}=\alpha_{C} \times \dot{m}_{f}^{*}
\end{gathered}
$$

It is worth noting that the calibration process is performed using the total amount of fuel injected from all six holes coupled with only the shape of the measured injection rate. The injection rate presented in the Results and Discussion section in $\mathrm{mg} / \mathrm{ms}$ represents the fuel injected from the entire six holes of the nozzle.

\section{Compensation for the outlet-target distance}

Since the sensor membrane is located at $4 \mathrm{~mm}$ away from the nozzle hole, it is therefore necessary to account for the time required for the spray plume to reach the membrane. The following equation was used to calculate the spray plume velocity and hence to compensate for the outlet-target distance.

$$
v=\frac{\dot{m}_{f}}{N_{h} \rho_{f} A_{h} C_{d}}
$$

Where,

$\boldsymbol{N}_{\boldsymbol{h}}$ - Number of holes in the injector nozzle,

$\boldsymbol{C}_{\boldsymbol{d}}$ - Discharge coefficient. 
In this analysis, the discharge coefficient was assumed to be 0.9 which is a typical value in high pressure diesel fuel injectors according to Desantes et al. [19]. As a result, the velocity is integrated and plotted as a function of time. Figure 5 illustrates the time required for the spray plume to travel from the nozzle hole to the sensor membrane. This velocity is integrated with respect to the time to get the actual distance. The time required to reach the membrane is a function of the injection rate as shown in equation 6 . To compensate for the traveling time, the injection rate was shifted accordingly.

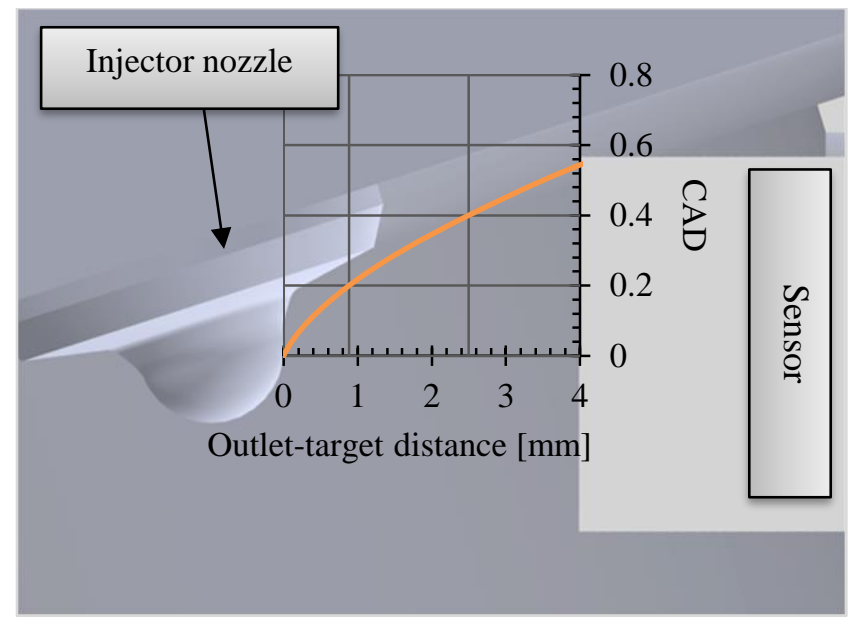

Figure 5. Estimation of the delay between the measured signal and the actual injection due to a finite distance between the sensing element and the injector nozzle [4].

\section{Results and Discussion}

The instantaneous impact force of a spray (momentum flux) over the entire injection process was recorded in a time-resolved domain and observed to undergo a gradual increase, reaching a peak when the needle reaches fully-open position [27]. It is worth mentioning that the impact force of a spray from one nozzle hole is somewhat similar to the impact forces from all other holes, according results obtained in [7]. Their results showed consistent trends with the spray impact forces of each nozzle hole. Thus, it is justified to process the injection rate measurement based on the impact force of a single spray from one hole and characterize the sum of the injection rates. It is noteworthy that the driving current does not immediately initiate the injections as there is a hydraulic delay which leads to a certain delay of the actual start of injection (SOI) as well as the end of injection (EOI). Hence, an effective SOI and EOI are herein defined. The effective SOI is defined as the time when the injection rate starts becomes non-zero. The effective EOI is defined as the time when the injection rate becomes zero again. Figure 6 provides a clear illustration of these terminologies.

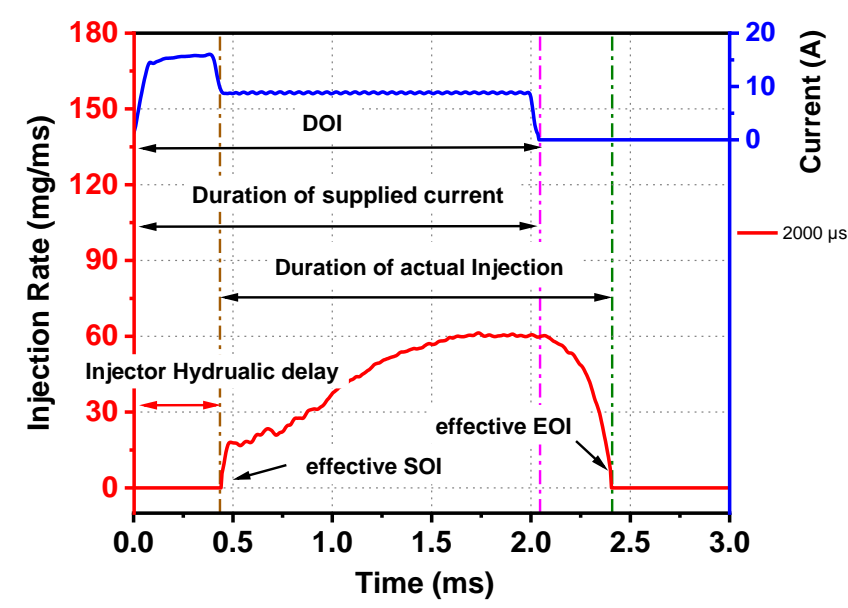

Figure 6. Terminologies used in the injection rate measurements.

\section{Injector hydraulic delay}

The injector hydraulic delay is the time interval between the start of the injector current and the effective SOI. In the split injection strategies, particularly when very short dwells are implemented and two injections merge, the injector hydraulic delay value of the second pulse is taken at the instant when the injection rate inflects and the slope becomes positive again. Figure 7 shows the injector hydraulic delay of the first injection events for the single injection cases as well as the split-injection strategies. It can be seen from Figures 7 and 8, that the increase in the injection pressures shortens the hydraulic delays significantly, in some cases by a more than a factor of two. The first injection of the split injection events is similar to the single injection cases since the SOI of the first injection was kept constant. In contrast, a more pronounced delays were noticed when dwell interval was kept long enough. It can be observed that the hydraulic delay decreases remarkably when dwell reduced. The lowest injector hydraulic delay is achieved in the range of $0.13 \mathrm{~ms}$ at the rail pressures of 1500 and 2000 bar with a dwell time of $100 \mu$ s.

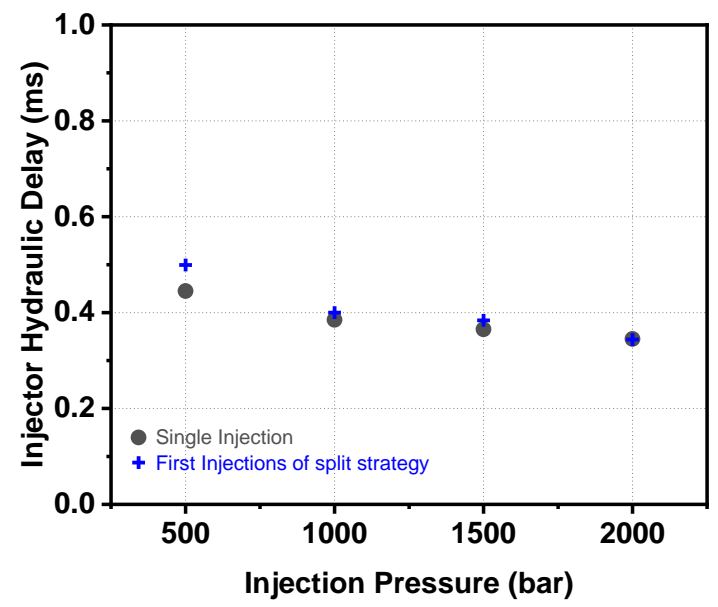

Figure 7. Injector hydraulic delay of the first injection events for single and split injections. 


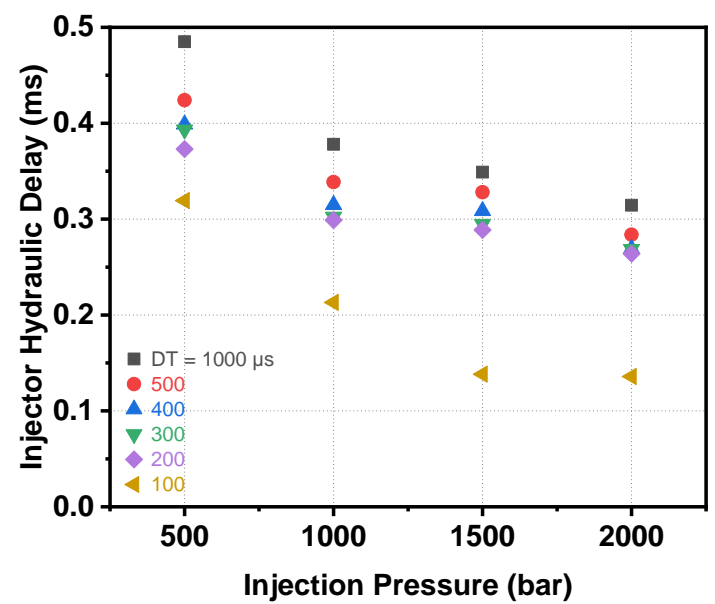

Figure 8. Effect of dwell time (DT) on the injector hydraulic delay of the second injection events for the split injection strategy.

\section{Single injection}

\section{Effect of Duration of Injection (DOI)}

A number of different injection durations is studied to ensure the consistency of the technique and to broaden the understanding of the behavior of a high flow rate solenoid injector used in the heavy-duty IC engine. The duration of the single injection is varied from 400 up to $2000 \mu \mathrm{s}$. The signal of the case with $400 \mu \mathrm{s}$ DOI and minimum injection pressure $\left(\mathrm{P}_{\mathrm{inj}}=500 \mathrm{bar}\right.$ ) was within the noise level of the acquisition system. The spray plume also had low momentum, hence that case could not be properly recorded and is not reported in the results.

In Figures 9-12, it can be observed that the small injection durations produce lower maximum injection rates. This is because it takes a considerable amount of time for the needle to reach its top position (fully open), and short injection durations limit the needle lift and, consequently, the fuel flow. Furthermore, as the injection pressure is increased, it can be observed that the maximum injection rate is achieved much earlier, which suggests that at higher pressures, the needle is moving faster. This shape change during injection process is quicker at higher injection pressures as seen in Figure 12. After the injection rate attained its maximum values, it becomes approximately quasi-steady. The decay of the injection rate at EOI is also slightly faster at higher injection pressures.

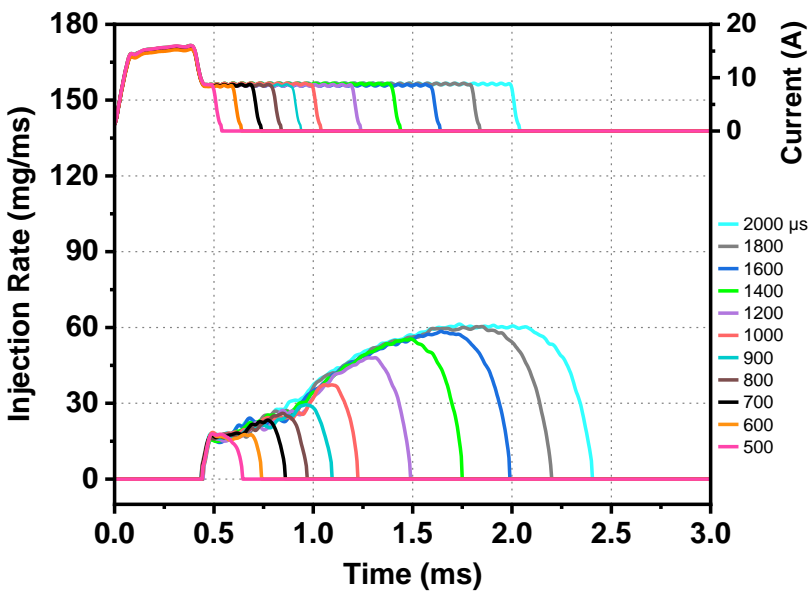

Figure 9. Comparison of the injection rates of different DOI ranging from 500 to $2000 \mu$ s at $\mathrm{P}_{\text {inj }}=500$ bar.

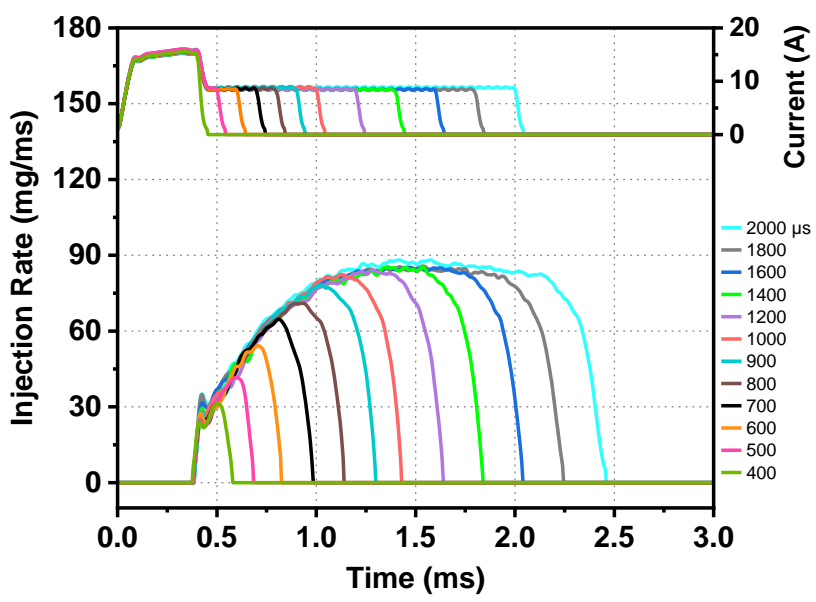

Figure 10. Comparison of the injection rates of different DOI ranging from 400 to $2000 \mu$ s at $P_{i n j}=1000$ bar.

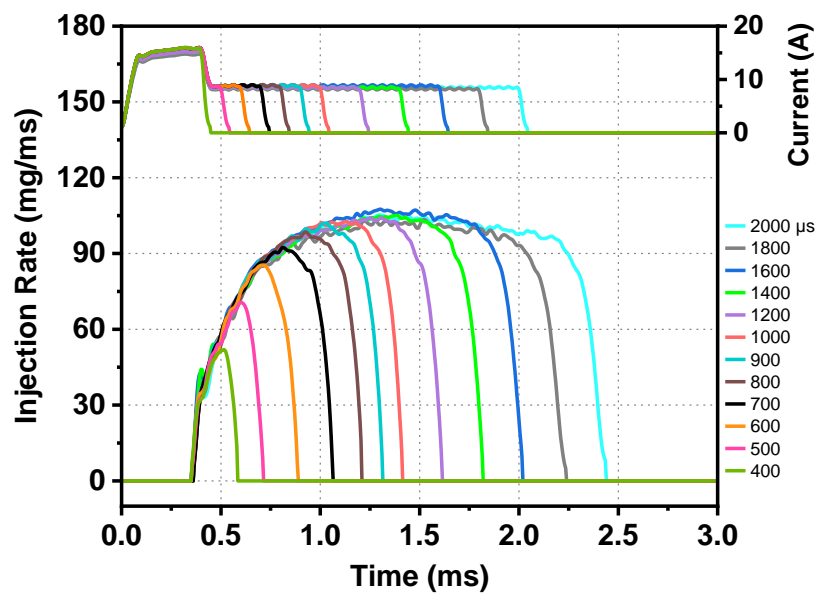

Figure 11. Comparison of the injection rates of different DOI ranging from 400 to $2000 \mu$ s at $P_{i n j}=1500$ bar. 


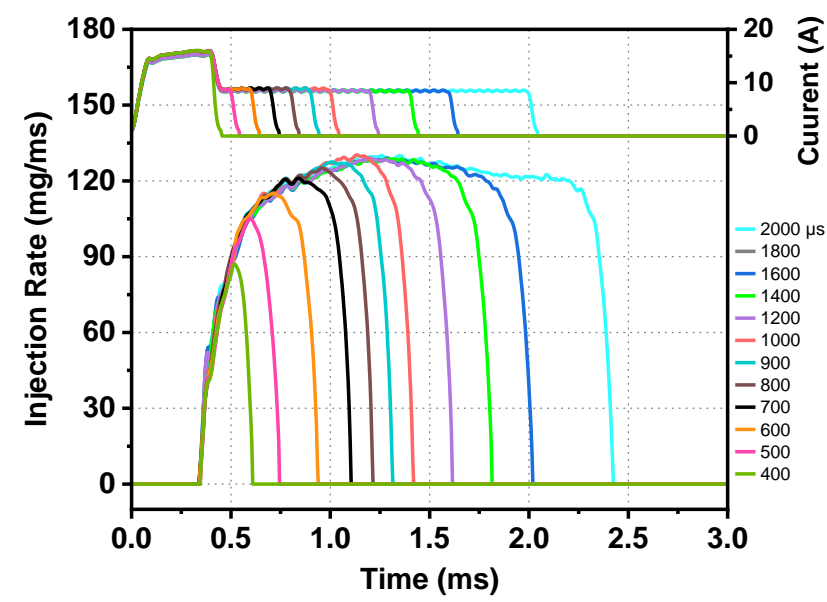

Figure 12. Comparison of the injection rates of different DOI ranging from 400 to $2000 \mu$ s at $P_{\text {inj }}=2000$ bar.

\section{Effect of the rail pressure on the injection rate}

The observations show the injection rate increases with the increase of the injection pressures due to the higher impact force of a penetrative spray. Figure 13 demonstrates the injection rate increase with the increase of the injection pressure. Furthermore, it can be observed that the SOI gradually advanced with higher injection pressure, which implies a reduction in the hydraulic delay. However, the effective EOI did not undergo significant change with varying pressure, neither did it change with varying DOI as seen in Figures 13 and 14. As a result, a higher amount of fuel total mass is recorded at higher injection pressures as shown in Figure 15. In addition, the fuel total mass injected in the DOI of $2000 \mu$ s case was increased by $97 \%$ compared to that $500 \mu \mathrm{s}$ DOI case at low $\mathrm{P}_{\text {inj }}=500$ bar. The increase in injection pressure up to 2000 bar significantly scaled up the fuel injected to approximately $87 \%$ more in the $2000 \mu$ s DOI case compared to $500 \mu \mathrm{s}$ DOI.

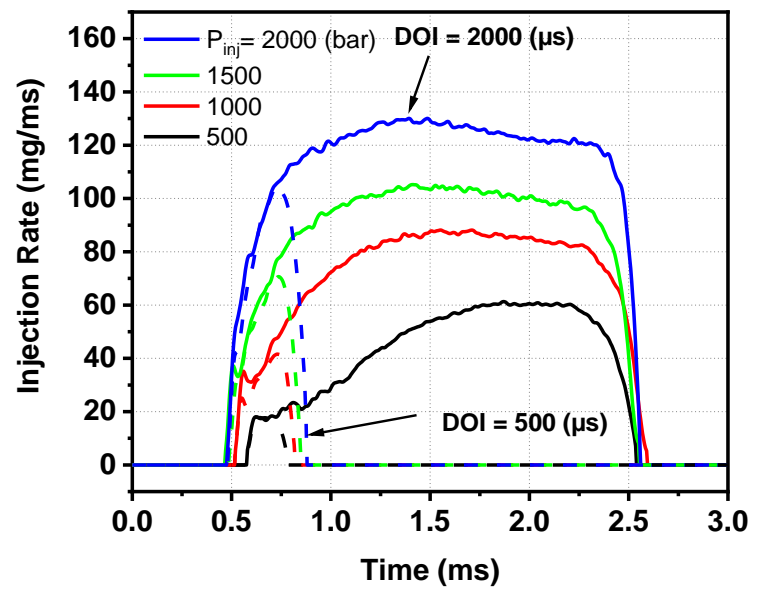

Figure 13. Effect of rail pressure for the DOI of $500 \mu$ s (dashed line) and 2000 $\mu \mathrm{s}$ (solid line).

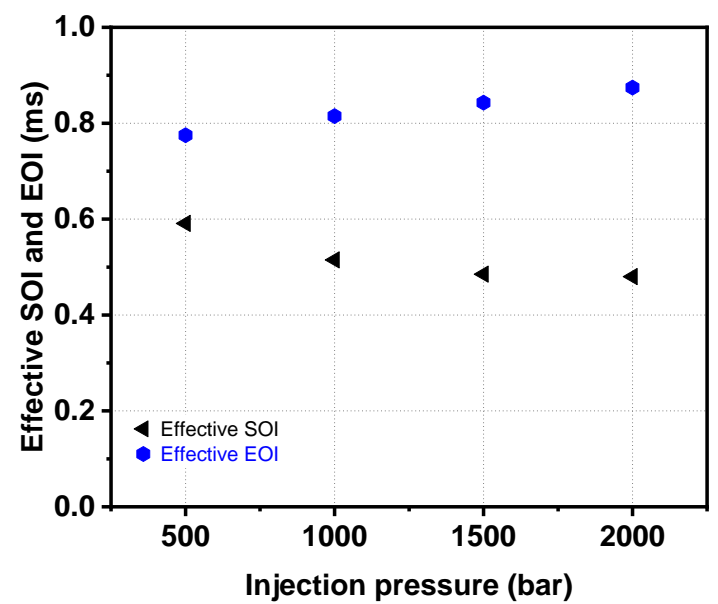

Figure 14. Effect of rail pressure on the effective SOI (black left triangle) and effective EOI (blue hexagon) at the DOI of $500 \mu$ s.

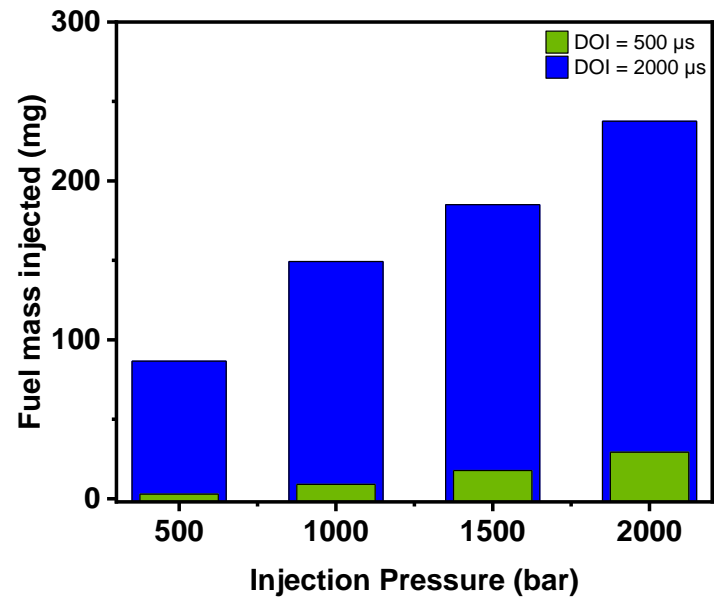

Figure 15. Effect of injection pressure on the total injected fuel quantity using the DOI of 500 and $2000 \mu \mathrm{s}$.

\section{Effect of the rail pressure fluctuation}

From Figure 13, it can be observed that the injection rate tends to decay slightly during the quasi-steady phase of the injection. This phenomenon is particularly apparent at long injection durations and can be explained by the drop in the rail pressure during the injection. Figure 16 illustrates the rail pressure as a function of time at different injection durations. The fluctuations of the common rail pressure in IC engines can significantly impact the injection rate profile. It can be seen that the rail pressure can drop by as much as $10 \%$ from the target pressure of 2000 bar at the DOI of $2000 \mu$ s. These rail fluctuations can be reduced by adapting the rail volume and the fuel pressurization strategy (or the control strategy of the injection system). In addition, the long injection durations seem to generate more adverse effects on the injection pulses. It should be noted that, this pressure drop is unique for the given set-up of the fuel injection system. 


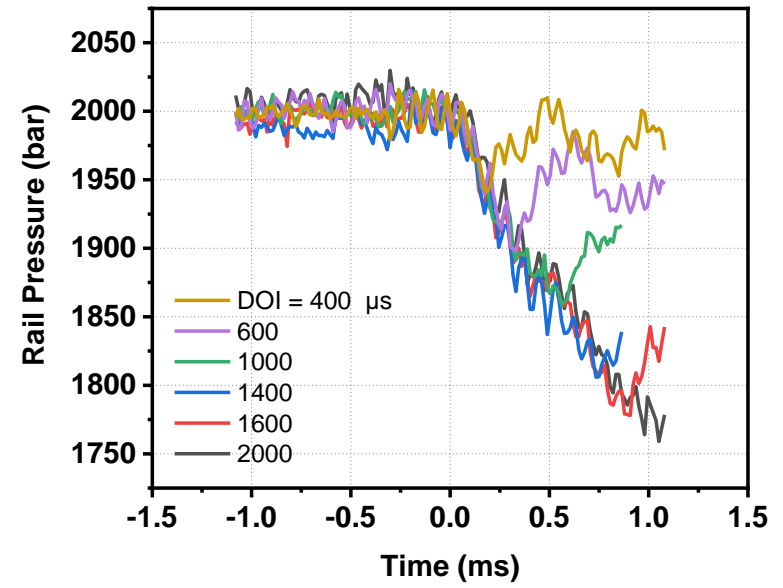

Figure 16. Drop of rail pressure at different duration of injection (DOI) for the case of $P_{\text {inj }}=2000$ bar.

\section{Double-split injection}

\section{Effect of dwell time (DT) on the split injection rate}

In the double-split injection strategy, the duration of each injection was fixed while the dwell time between the injections was varied to study the dynamic behavior of the injector in the pressure range of 500-2000 bar. As seen in Figure 17, the injection rate shapes are similar at low injection pressures regardless of the DT implemented. However, it can be observed in Figures 18, 19, and 20 that at rail pressures exceeding 1000 bar the change of the dwell time (DT) has a dramatic effect on the second injection rate shape. Decreasing the dwell time leads to a rise of the rate of the second injection. This results in a higher total amount of fuel injected as will be discussed later. However, dwell times longer than $500 \mu$ s lead to similar injection rate shape for the second injection.

The injector was also characterized utilizing dwell times that are shorter than the values recommended by the manufacturer $(<200 \mu \mathrm{s})$ [1]. As can be seen from Figure 19, when the rail pressure is 1500 bar, dwell times equal or lower than $100 \mu$ s lead to a merger of the two injections. As the rail pressure is further increased, this phenomenon becomes even more pronounced. This is mainly owing to the significantly reduced hydraulic delay at higher injection pressures, as discussed earlier. Since the timing of the second injection is kept constant, shorter hydraulic delay leads to earlier start of injection and, in some cases, the merger of the injections. Generally, very short dwell times $(<200 \mu \mathrm{s})$ have adverse effects on the injection system stability, in some cases even leading to a complete loss of pressure in the rail. This minimum dwell time is injector-specific and a different diesel injector might be able to sustain shorter dwells, as reported in [27]. For this specific injector, the minimum dwell time requires a shot-to-shot separation to be within 1.5 CAD (at $1200 \mathrm{RPM}$ ) for double injection strategy. In contrast, the minimum dwell time is recommended to be $100 \mu$ s corresponding to approximately $0.8 \mathrm{CAD}$ for the rail pressures below 1000 bar at the same engine speed.

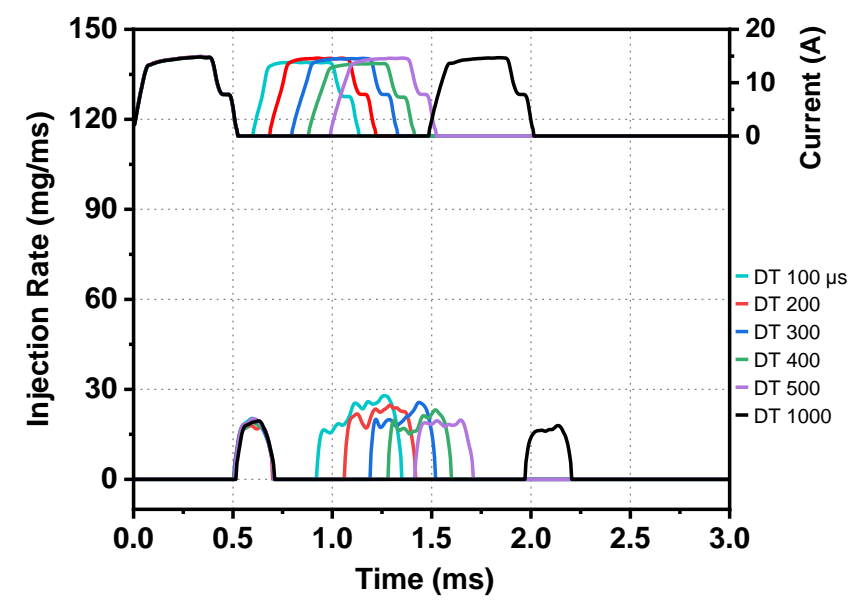

Figure 17. Effect of the dwell time (DT) on the rate of double-split injection at $P_{\text {inj }}=500$ bar.

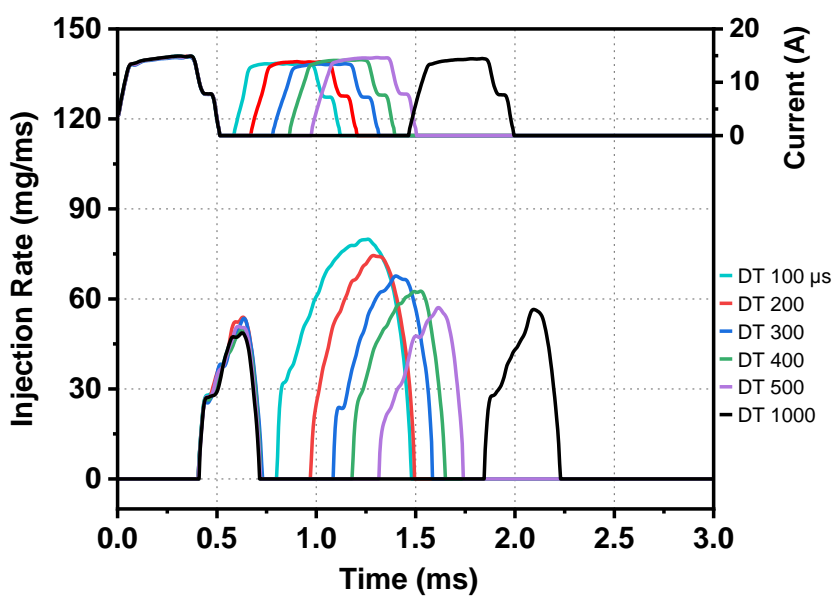

Figure 18. Effect of the dwell time (DT) on the rate of double-split injection at $P_{\text {inj }}=1000$ bar.

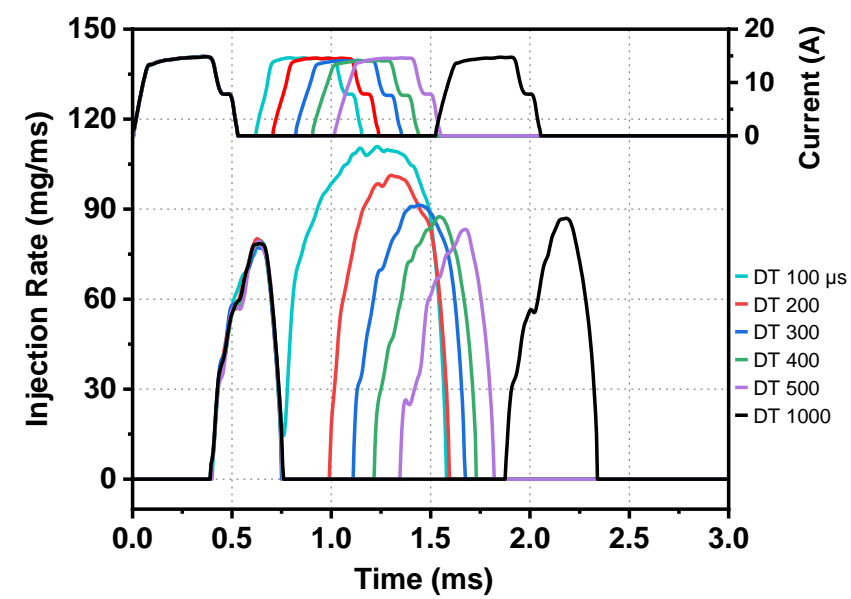

Figure 19. Effect of the dwell time (DT) on the rate of double-split injection at $P_{\text {inj }}=1500 \mathrm{bar}$ 


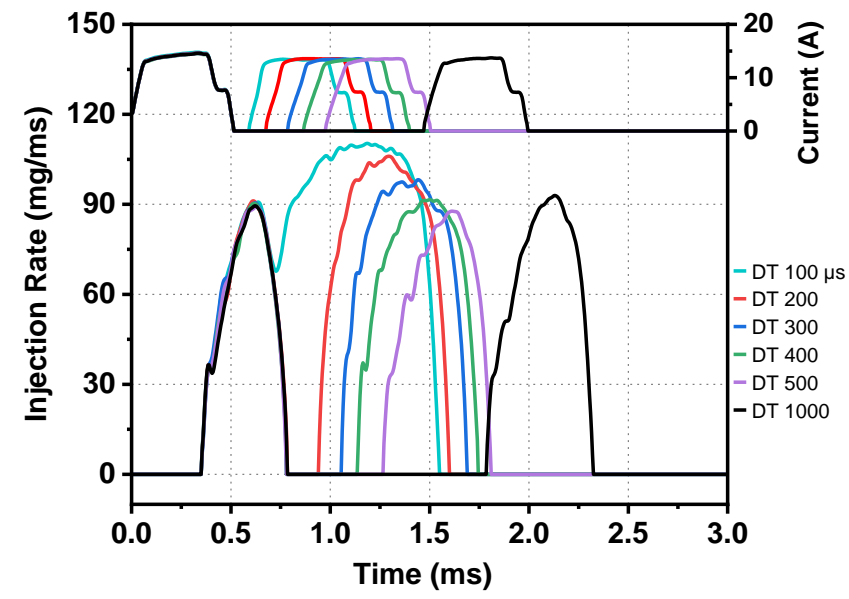

Figure 20. Effect of the dwell time (DT) on the rate of double-split injection at $P_{\text {inj }}=2000 \mathrm{bar}$.

\section{Effect of rail pressure on the double-split injection}

As discussed in the previous subsection, rail pressure has a significant effect on the hydraulic delay of the injector. In Figures 21 and 22, the injection rate of the double-split injection is presented as a function of the rail pressure at the constant dwell time of 100 and $1000 \mu \mathrm{s}$, respectively. It can be seen that as the rail pressure is increased, the SOI of both injections advance considerably, even leading to a merger of the two injections at the rail pressures higher or equal to 1500 bar. The merger happens because the needle cannot reach its fully closed position before the subsequent needle-lift is initiated. This also leads to significantly higher total amount of fuel injected. In the case of 1000 $\mu \mathrm{s}$, both injection rates peak at $\sim 90(\mathrm{mg} / \mathrm{ms})$ whereas the dwell of 100 $\mu$ s leads to $\sim 110(\mathrm{mg} / \mathrm{ms})$ in the second injection. The effect of the rail pressure on the EOI timing is, however, more ambiguous. First the needle closing time increased with the pressure ranging from 500 bar up to 1500 bar, however, a further increase in rail pressure led to a quicker closing of the needle.

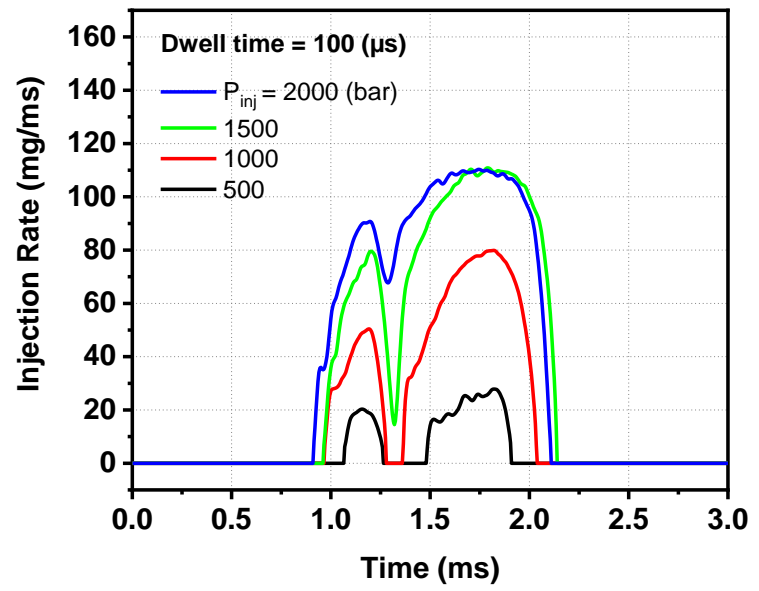

Figure 21. Effect of rail pressures on the injection rates of split-injection at 100 us dwell time (DT)

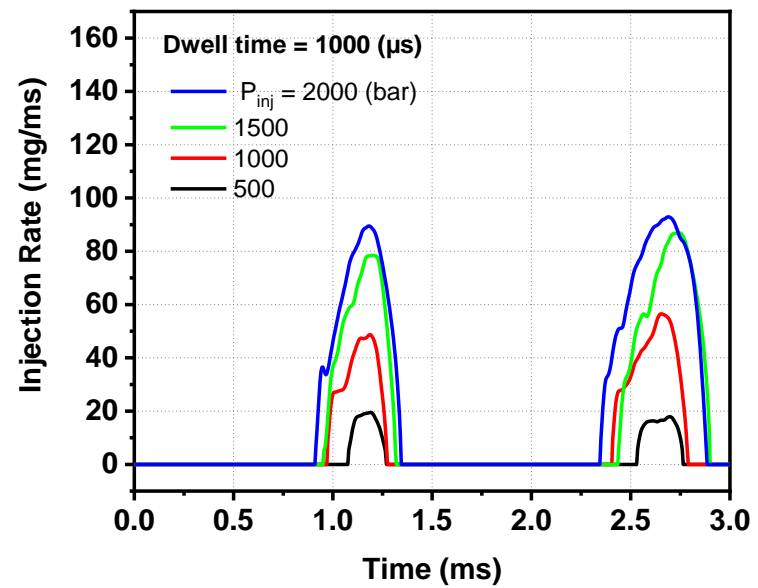

Figure 22. Effect of rail pressures on the injection rates of split-injection at $1000 \mu$ s dwell time (DT)

\section{Effect of dwell time (DT) on the total fuel mass injected}

Figures 23 and 24 illustrate the dwell time effects on the total fuel mass injected at the fixed DOI of $500 \mu \mathrm{s}$. Longer dwell times lead to approximately the same fuel quantity injected during the second injection. In contrast, and regardless of the second DOI, a dramatic increase in the accumulated fuel mass of the second injection is observed with shorter dwell times. The case with DT $=100 \mu$ s and $P_{i n j}$ $=500$ bar shows approximately $74 \%$ of the fuel total mass was injected in the second DOI, whereas only $54 \%$ of the fuel total mass was injected when dwell was increased to $1000 \mu$ s. Similarly, $72 \%$ of fuel total mass was observed in the $100 \mu \mathrm{s}$ dwell compared to $58 \%$ of the fuel total at $1000 \mu \mathrm{s}$ dwell when injection pressure was increased to 2000 bar. Slight variations of fuel masses injected in the first injection are most likely a result of cycle-to-cycle variations.

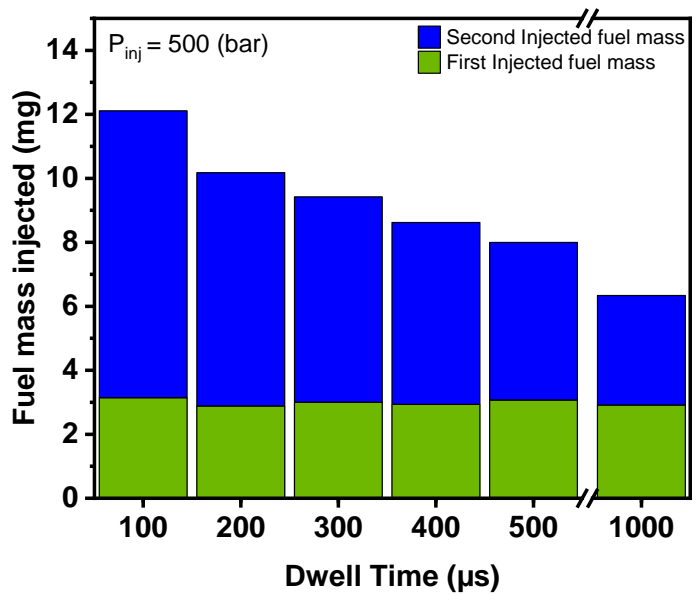

Figure 23. Effect of dwell time (DT) on the total injected fuel quantity using the DOI of $500 \mu$ s at $P_{i n j}=500 \mathrm{bar}$ 


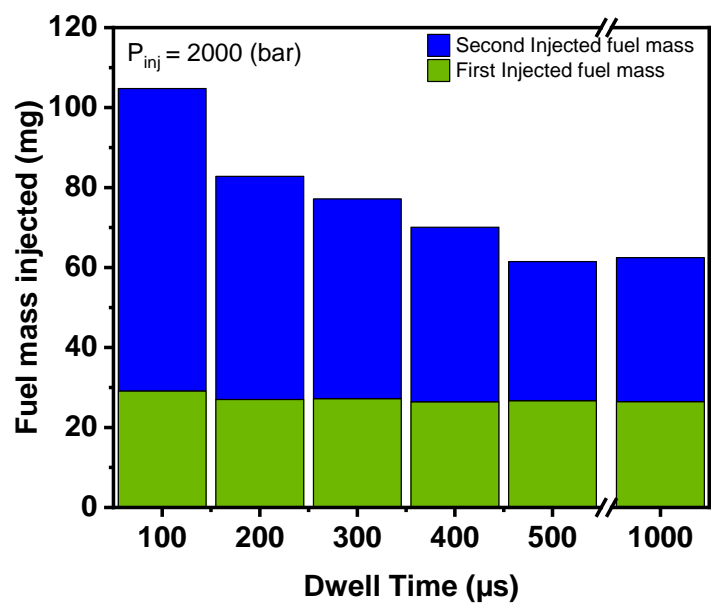

Figure 24. Effect of dwell time (DT) on the total injected fuel quantity using the DOI of $500 \mu$ s at $P_{i n j}=2000$ bar.

\section{Multi-split injection}

One of the limitations of the injection rate measurement method presented in this work is its inability to record very short injection events. Figure 25 shows the injector current and the measured injection rate as function of time for a five-injection strategy required to achieve isobaric combustion at loads close to 20 bar IMEPg. The injection rates were successfully measured using this method for all injections events with durations longer than $200 \mu$ s. However, the spray generated by the first pulse could not be captured with the current setup. Its duration was set at $190 \mu \mathrm{s}$. The main purpose of this injection was to create favorable in-cylinder conditions for immediate ignition of the subsequent sprays. However, due to its negligible momentum, this first pilot injection did not exert enough force on the transducer, hence no signal was obtained.

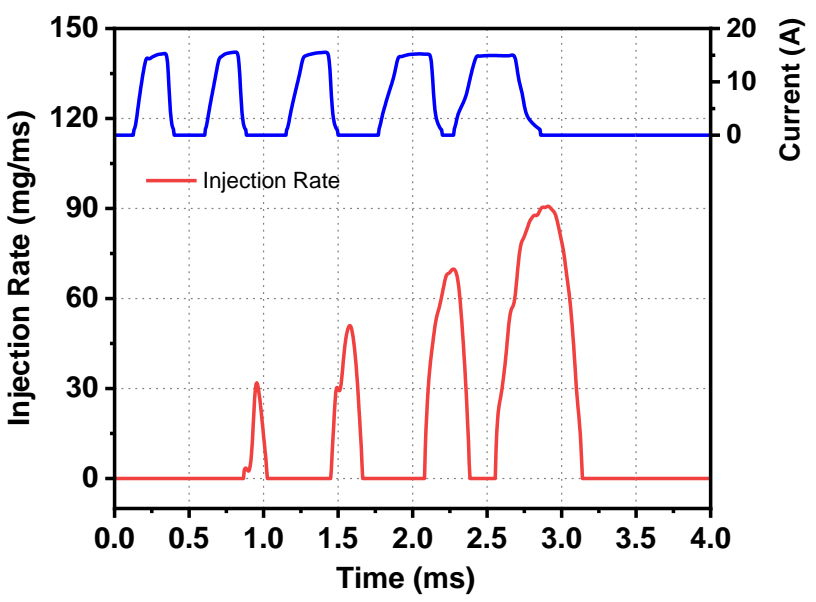

Figure 25. Injection rate and current of five-injection strategy to achieve constant-pressure combustion on the same engine configuration at $P_{\text {inj }}=$ 2300 bar

\section{Summary/Conclusions}

In-situ injection rate measurements were conducted on a modified Volvo HD Diesel engine. Injector characterization setup was installed directly on the engine after removing the piston and the liner. Analysis of the effective start and end of injection timings, injection rate shape, dwell time, and total fuel quantity were performed on various single and double-injection strategies. According to the experimental findings, the following conclusions have been drawn:

1. In-situ injection rate characterization was demonstrated and allowed measurement at more realistic engine operating conditions in terms of acoustics and fuel temperature.

2. In spray momentum technique, the post-processing of the signal was demonstrated to be key factor to achieve good quality measurements. The charge leak from the amplifier and the effective SOI were corrected and this allowed a better estimation of the hydraulic delays.

3. The fluctuation and the drop of rail pressure were very specific to the pumping system used. Direct correlation between the drop in pressure and the injection rate shape was demonstrated, particularly at long DOI. The injection rate did not have a square shape and the mass flow was decreasing before the EOI.

4. Injection rate shape of single injections is highly dominated by the injection pressure. The response of the needle to the signal to initiate the flow is significantly faster at higher injection pressures. On the other hand, the needle closing timing was not clearly correlated to the changing pressures.

5. Injector hydraulic delay becomes shorter as the dwell interval is reduced.

6. The changes in the dwell time between two injections have profound effects on the rate shape of the latter injection. Decreasing the dwell time by a factor of 10 usually leads to an increase in the amount of fuel injected in the second shot by a factor of 2 for the tested injector.

\section{References}

1. Meek, George A., Ryan Williams, Dan Thornton, Patrick Knapp, and Stephen Cosser. F2E-Ultra High Pressure Distributed Pump Common Rail System. No. 2014-01-1440. SAE Technical Paper, 2014.

2. Nordgren, Henrik, Anders Hultqvist, and Bengt Johansson. Start of injection strategies for HCCI-combustion. No. 2004-01-2990. SAE Technical Paper, 2004.

3. Nehmer, Daniel A., and Rolf D. Reitz. "Measurement of the effect of injection rate and split injections on diesel engine soot and NOx emissions." SAE transactions (1994): 1030-1041.

4. Babayev, Rafig, Moez Ben Houidi, Vijai Bhavani Shankar, Bassam S. E. Aljohani, and Bengt Johansson. "Injection Strategies for Isobaric Combustion”, JSAE paper no: 20199124, SAE Technical Paper, 2019.

5. Babayev, Rafig, Moez Ben Houidi, Arne Andersson, and Bengt Johansson. Isobaric Combustion: A Potential Path to High Efficiency, in Combination with the Double Compression Expansion Engine (DCEE) Concept. No. 2019-01-0085. SAE Technical Paper, 2019. 
6. Wang, Ziman, Miroslaw L. Wyszynski, Hongming Xu, Nik Rosli Abdullah, and Jakub Piaszyk. "Fuel injection and combustion study by the combination of mass flow rate and heat release rate with single and multiple injection strategies." Fuel Processing Technology 132 (2015): 118-132.

7. Luo, Fuqiang, Huifeng Cui, and Shaofeng Dong. "Transient measuring method for injection rate of each nozzle hole based on spray momentum flux." Fuel 125 (2014): 20-29.

8. Bosch, Wilhelm. The fuel rate indicator: a new measuring instrument for display of the characteristics of individual injection. No. 660749. SAE Technical Paper, 1966.

9. Ishikawa, Satoshi, Yukimitsu Ohmori, Susumu Fukushima, Takao Suzuki, Akio Takamura, and Takeyuki Kamimoto. Measurement of rate of multiple-injection in CDI diesel engines. No. 2000-01-1257. SAE Technical Paper, 2000.

10. Postrioti, Lucio, Giacomo Buitoni, Francesco C. Pesce, and Claudio Ciaravino. "Zeuch method-based injection rate analysis of a common-rail system operated with advanced injection strategies." Fuel 128 (2014): 188-198.

11. Arcoumanis, Constantine, M. S. Baniasad, and M. S. Banias. "Analysis of consecutive fuel injection rate signals obtained by the Zeuch and Bosch methods." SAE Transactions (1993): 13711384.

12. Payri, R., F. J. Salvador, J. Gimeno, and G. Bracho. "A new methodology for correcting the signal cumulative phenomenon on injection rate measurements." Experimental techniques 32, no. 1 (2008): 46-49.

13. Matsuoka, S., K. Yokota, and T. Kamimoto. "The measurement of injection rate." In Proceedings of the Institution of Mechanical Engineers, Conference Proceedings, vol. 184, no. 10, pp. 87-94. Sage UK: London, England: SAGE Publications, 1969.

14. Bopp, S., F. Durst, J. Holweg, and H. Weber. "A laser-Doppler sensor for flowrate measurements." Flow Measurement and Instrumentation 1, no. 1 (1989): 31-38.

15. Zeng, Wei, Min Xu, Ming Zhang, Yuyin Zhang, and David J. Cleary. "Macroscopic characteristics for direct-injection multihole sprays using dimensionless analysis." Experimental thermal and fluid science 40 (2012): 81-92.

16. Marčič, Milan. "A new method for measuring fuel-injection rate." Flow Measurement and Instrumentation 10, no. 3 (1999): 159165.

17. Payri, F., R. Payri, F. J. Salvador, and J. Gimeno. "Comparison between different hole to hole measurement techniques in a diesel injection nozzle." SAE transactions (2005): 1396-1403.

18. Payri, R., J. M. Garcia, F. J. Salvador, and Jaime Gimeno. "Using spray momentum flux measurements to understand the influence of diesel nozzle geometry on spray characteristics." Fuel 84, no. 5 (2005): 551-561.

19. Desantes, José M., J. Javier López, Marcos Carreres, and Darío López-Pintor. "Characterization and prediction of the discharge coefficient of non-cavitating diesel injection nozzles." Fuel 184 (2016): 371-381.

20. Manin, Julien, Alan Kastengren, and Raul Payri. "Understanding the acoustic oscillations observed in the injection rate of a common-rail direct injection diesel injector." Journal of engineering for gas turbines and power 134, no. 12 (2012): 122801

21. AlRamadan, Abdullah S., Moez Ben Houidi, Bassam SE Aljohani, Hassan Eid, and Bengt Johansson. Compression Ratio and Intake Air Temperature Effect on the Fuel Flexibility of Compression Ignition Engine. No. 2019-24-0110. SAE Technical Paper, 2019.

22. Payri, Raul, Antonio García, Vicent Domenech, Russell Durrett, and Alejandro H. Plazas. "An experimental study of gasoline effects on injection rate, momentum flux and spray characteristics

Page 11 of 12 using a common rail diesel injection system." Fuel 97 (2012): 390-399.

23. Lundgren, Marcus, Joakim Rosell, Mattias Richter, Öivind Andersson, Bengt Johansson, Andersson Arne, and Marcus Alden. Optical study on combustion transition from HCCI to PPC with gasoline compression ignition in a HD engine. No. 2016-010768. SAE Technical Paper, 2016.

24. Alfuso, S., L. Allocca, M. Auriemma, G. Caputo, F. E. Corcione, A. Montanaro, and G. Valentino. Analysis of a high pressure diesel spray at high pressure and temperature environment conditions. No. 2005-01-1239. SAE Technical Paper, 2005.

25. Lucchini, Tommaso, Gianluca D'Errico, Daniele Ettorre, Federico Brusiani, Gian Marco Bianchi, Alessandro Montanaro, and Luigi Allocca. Experimental and numerical investigation of high-pressure diesel sprays with multiple injections at engine conditions. No. 2010-01-0179. SAE Technical Paper, 2010.

26. Mohan, Balaji, Wenming Yang, Kun Lin Tay, and Wenbin Yu. "Macroscopic spray characterization under high ambient density conditions." Experimental Thermal and Fluid Science 59 (2014): 109-117.

27. Sangiah, D. K., and L. C. Ganippa. "Application of spray impingement technique for characterisation of high pressure sprays from multi-hole diesel nozzles." International Journal of Thermal Sciences 49, no. 2 (2010): 409-417.

28. Ehleskog, Rickard, Valeri Golovitchev, Ingemar Denbratt, Sven Andersson, and Carlo Alberto Rinaldini. Experimental and numerical investigation of split injections at low load in an HDDI diesel engine equipped with a piezo injector. No. 2006-01-3433. SAE Technical Paper, 2006.

\section{Contact Information}

Bassam S. E. Aljohani

Clean Combustion Research Center

King Abdullah University of Science and Technology

Al-Kindi Building (5), Level 4, 4315-WS08

Thuwal 23955-6900, Saudi Arabia

T +966 (0) 12808 7846, M +966 (0) 549001213

Email: bassam.aljohani.1@kaust.edu.sa

\section{Acknowledgment}

The authors would like to thank Dr. Balaji Mohan for his considerable cooperation and valuable feedback throughout the progress of the experiment. Special thanks to the Engine group for their contribution during engine operation. 


\section{Definitions/Abbreviations}

IC

DOI

HR

NOx

PM

HCCI

SOHC

CAD

LP

TDC

FFT

FIR

SOI

EOI

DT

IMEPg
Internal combustion.

Duration of injection.

Heat release.

Nitric oxide and oxide of nitrogen.

Particulate matters.

Homogenous charge compression ignition.

Single overhead camshaft.

Crank angle degree.

Low pass.

Top dead center.

Fast Fourier transform.

Finite impulse response.

Start of injection.

End of injection.

Dwell time.

Gross indicated mean effective pressure. 